\title{
Effectiveness of transcranial direct current stimulation alone or associated with antidepressants, psychotherapy or cognitive therapies for the management of major depression - a review on clinical trials
}

Eficácia da estimulação transcraniana por corrente contínua isolada ou associada a antidepressivos, psicoterapia ou terapias cognitivas para o tratamento da depressão maior - uma revisão de ensaios clínicos

Efectividad de la estimulación transcraneal con corriente directa sola o en combinación con antidepresivos, psicoterapia o terapias cognitivas para el tratamiento de la depresión mayor - una revisión de ensayos clínicos

Received: 11/04/2021 | Reviewed: 11/15/2021 | Accept: 11/22/2021| Published: 12/01/2021

João Márcio Borgue de Oliveira ORCID: https://orcid.org/0000-0003-3670-8476 Federal University of Alfenas, Brazil E-mail: joaom_borgue@hotmail.com

Thaís Hernandes Salmaso ORCID: https://orcid.org/0000-0003-0214-7834 Federal University of Alfenas, Brazil E-mail: thaishsalmaso@gmail.comr

Edvaldo José Rodrigues Cardoso ORCID: https://orcid.org/0000-0002-9701-450X Federal University of Alfenas, Brazil

E-mail: edvaldo.cardoso@unifal-mg.edu.br

Silvia Graciela Ruginsk

ORCID: https://orcid.org/0000-0002-0534-0077 Federal University of Alfenas, Brazil E-mail: silvia.leitao@unifal-mg.edu.br

\begin{abstract}
Considering the evidence that transcranial direct current stimulation (TDCS) is effective for major depression (MD) management, and the perspective of including this therapeutic modality among conventional treatments, our main objective was to evaluate the reported effectiveness of TDCS, combined with antidepressants, psychotherapy or cognitive therapies, on clinical and neuropsychological outcomes in MD patients. We performed an integrative review of clinical trials, guided by the PICO strategy, using PubMed and Ovid MEDLINE $§$ databases. Twelve scientific reports were selected according to inclusion and exclusion criteria, comparatively analyzed, discussed and theorized. According to these studies, anodic TDCS applied to the left dorsolateral prefrontal cortex exhibits significant antidepressant effects, comparable to low dose sertraline $(50 \mathrm{mg} / \mathrm{day})$. In addition, two main studies indicate that antidepressant effects can be enhanced by combined treatments, especially TDCS + sertraline. There is also evidence of a synergistic effect of TDCS and cognitive control training, although this finding is not unanimous under different methodological approaches, thus requiring further studies in order to support the correlation between neurocognitive and clinical improvement with this type of combined therapy. Finally, TCDS-induced positive cognitive effects, mainly on working memory, were also verified. In conclusion, the small number of reports employing TDCS associated with other therapies show very discrepant research methods, as well as short follow-up periods, which together with low sample number, leave many knowledge gaps to be answered by future studies.
\end{abstract}

Keywords: Depressive disorder; Transcranial direct current stimulation; Combined modality therapy.

\section{Resumo}

Considerando as evidências de que a estimulação transcraniana por corrente contínua (ETCC) é eficaz para o manejo da depressão maior (DM) e a perspectiva de incluir essa modalidade terapêutica entre os tratamentos convencionais, nosso principal objetivo foi avaliar a eficácia relatada da ETCC, combinada com antidepressivos, psicoterapia ou terapias cognitivas, em resultados clínicos e neuropsicológicos em pacientes com DM. Realizamos uma revisão integrativa de ensaios clínicos, orientada pela estratégia PICO, utilizando as bases de dados PubMed e Ovid MEDLINE®. Doze trabalhos científicos foram selecionados de acordo com critérios de inclusão e exclusão, analisados 
comparativamente, discutidos e teorizados. De acordo com esses estudos, a ETCC anódica aplicada ao córtex pré-frontal dorsolateral esquerdo exibe efeitos antidepressivos significativos, comparáveis à sertralina em baixas doses (50 mg/dia). Além disso, dois estudos principais indicam que os efeitos antidepressivos podem ser aumentados por tratamentos combinados, especialmente ETCC + sertralina. Também há evidências de um efeito sinérgico da ETCC e do treinamento de controle cognitivo, embora esse achado não seja unânime em diferentes abordagens metodológicas, e, portanto, requeira mais estudos para fundamentar a correlação entre a melhora neurocognitiva e clínica com esse tipo de terapia combinada. Por fim, efeitos cognitivos positivos induzidos por ETCC, principalmente na memória de trabalho, também foram verificados. Em conclusão, o pequeno número de trabalhos que empregam o ETCC associado a outras terapias mostra métodos de pesquisa muito discrepantes, bem como curtos períodos de acompanhamento, que, aliados ao baixo número de amostra, deixam muitas lacunas de conhecimento a serem respondidas em estudos futuros.

Palavras-chave: Transtorno depressivo; Estimulação transcraniana por corrente continua; Terapia combinada.

\section{Resumen}

Considerando la evidencia de que la estimulación transcraneal de corriente directa (ETCD) es efectiva para el manejo de la depresión mayor (DM) y la perspectiva de incluir esta modalidad terapéutica entre los tratamientos convencionales, nuestro principal objetivo fue evaluar la efectividad reportada de ETCD, combinada con antidepresivos, psicoterapia o terapias cognitivas, sobre los resultados clínicos y neuropsicológicos en pacientes con DM. Realizamos una revisión integradora de ensayos clínicos, guiados por la estrategia PICO, utilizando las bases de datos PubMed y Ovid MEDLINE®. Se seleccionaron doce trabajos científicos según criterios de inclusión y exclusión, analizados comparativamente, discutidos y teorizados. Según estos estudios, la tDCS anódica aplicada a la corteza prefrontal dorsolateral izquierda exhibe efectos antidepresivos significativos, comparables a la sertralina en dosis bajas (50 $\mathrm{mg} /$ día). Además, dos estudios principales indican que los efectos antidepresivos pueden incrementarse mediante tratamientos combinados, especialmente ETCD + sertralina. También hay evidencia de un efecto sinérgico de la tDCS y el entrenamiento de control cognitivo, aunque este hallazgo no es unánime en los diferentes enfoques metodológicos y, por lo tanto, requiere más estudios para corroborar la correlación entre la mejoría neurocognitiva y clínica con este tipo de terapia combinada. Finalmente, también se verificaron los efectos cognitivos positivos inducidos por ETCD, principalmente sobre la memoria de trabajo. En conclusión, el escaso número de estudios que utilizan ETCD asociado a otras terapias muestra métodos de investigación muy discrepantes, así como períodos de seguimiento cortos, que, combinados con el reducido tamaño de la muestra, dejan muchas lagunas de conocimiento por resolver en futuros estudios.

Palabras clave: Transtono depresivo; Estimulación transcraneal de corriente directa; Terapia combinada.

\section{Introduction}

Depressive disorders are characterized by a sad, irritable and empty mood, accompanied by cognitive and somatic changes that affect social, professional and other important individual aspects. The main relevant disorder in this group is major depression (MD) (Association, 2014).

Between 2005 and 2015, the number of people affected by MD increased by 18\%, with approximately 322 million people worldwide currently living with this mental disorder (Depression-Global-Health-Estimates @Www.Who.Int, n.d.). Mental disorders correspond to $22.9 \%$ of the years lived with disability (Years Lived with Disability - YLD) and $7.4 \%$ of the years adjusted for disability (Disability Adjusted Life Year - DALY), of which 40.5\% correspond to depressive disorders (Berti de Azevedo Barros et al., 2016).

Depressive disorders, especially MD, have multiple causal and maintenance variables, reflecting its complexity and the varied responses to conventional treatment. The determining factors include genetic, psychological or cognitive profile, lifestyle, previous stress or trauma, inflammation and hyperactivity of the hypothalamic-pituitary-adrenal axis, and altered neuroplasticity and neurogenesis. All of these aspects can interact with each other, leading to a positive feedback, generating the classic clinical phenotype (Dean \& Keshavan, 2017; Kim \& Won, 2017).

For MD treatment, pharmacological and non-pharmacological methods can be used. Pharmacotherapy includes antidepressant drugs based on multiple mechanisms of action, side effects and particularities (Hillhouse \& Porter, 2015). The main antidepressant effects are achieved by the potentiation of monoaminergic transmission and production of neurotrophic factors, with the respective positive actions on neuroplasticity and neurogenesis (Willner et al., 2013). Besides, psychotherapies are widely used as a non-pharmacological approach, particularly the cognitive-behavioral therapy (CBT), due to greater evidence 
of improvement (Cuijpers et al., 2013; López-López et al., 2019; Santoft et al., 2019; Zakhour et al., 2020). However, many patients with severe MD (i.e. with psychotic depression and under emergencies) may experience refractoriness to conventional drug treatment, being electroconvulsive therapy an alternative to be considered. Although it exhibits good effectiveness, is fast and safe, it is associated with high rates of relapse (Çakir \& Çağlar, 2017; Hermida et al., 2018; Swedish Council on Health Technology Assessment, 2004).

Cognitive therapies, such as cognitive control training (CCT), work with specific neuropsychological aspects, such as working memory and attention, and also have antidepressant efficacy, although research is still heterogeneous and emerging, requiring careful analysis. The correlation between $\mathrm{MD}$ and cognition has been increasingly demonstrated, so that changes in cognitive control are related to increased risk of MD development (Koster et al., 2017). As extensively demonstrated, MD patients focus their attention to emotionally negative information arising from both external and internal stimuli, resulting in greater emphasis on thoughts related to negative beliefs or representations and depressive ruminations (Koster et al., 2017; Mennen et al., 2019).

Neuromodulation also constitutes a non-pharmacological modality for the treatment of neurological and psychiatric disorders. This approach is based on the stimulation and / or inhibition of the activity of one or more brain regions and neuronal circuits (Edwards et al., 2017). Transcranial direct current stimulation (TDCS) is a non-invasive neuromodulation modality whose main effect is a change in the resting potential of the neuronal membrane, which can become more excitable (anodic stimulation) or less excitable (cathodic stimulation) (Lefaucheur et al., 2017). It employs two electrodes, the anode (positive) and the cathode (negative), arranged on the scalp above different brain regions varying according to treatment protocols. In such an arrangement, a low amperage direct current (between 0.15 to $2 \mathrm{~mA}$ ) is applied for 10 to 20 minutes per session (Arul-Anandam \& Loo, 2009).

The most used TDCS approach for MD management is a bifrontal mounting, with the anode set over F3 region (left dorsolateral prefrontal cortex - DLPFC), in order to increase its activity, and the cathode set over F4 region (right DLPFC), aiming a reduced activity of this area (Lefaucheur et al., 2017). This arrangement is based on studies that suggest an asymmetric function between the two sides of prefrontal cortex in MD patients, with left DLPFC showing underactivity and/or right DLPFC exhibiting overactivity. This particular feature shows increased tendency to balance in response to treatment (Grimm et al., 2008; Kocmur et al., 1998; Mottaghy et al., 2002), presumably reducing overall left prefrontal activity (Bruder et al., 2017).

Computational and pre-clinical studies show that not only the areas submitted to TDCS that are influenced by this technique, but also adjacent brain structures and related neural networks (Csifcsák et al., 2018; Rahman et al., 2017). Brain activity can also amplify the TDCS-generated electric fields, since postsynaptic depolarization and trigger probability is increased by the presynaptic firing rate or synchronized activation of presynaptic cells, in a cooperative operation between directly stimulated target regions and adjacent connections, also influenced by TCDS-induced diffused current (Rahman et al., 2017).

Although the study conducted by Brunoni and colleagues in 2017 (Andre R Brunoni et al., 2017) lacked a group combining active TDCS + pharmacotherapy, TDCS was significantly more effective than placebo, but less effective than escitalopram, in improving clinical variables in MD patients. In this report, 202 adult depressive individuals were divided into three groups: Group 1 - active TDCS + oral placebo; Group 2 - TDCS simulation + escitalopram; Group 3 - TDCS simulation + oral placebo. The patients received two-stage 30-minute sessions of pre-frontal stimulations of $2 \mathrm{~mA}$ with anode and cathode positions set at F3 and F4 regions, respectively. Initially, applications were daily for the first 15 business days, and then continued for 7 weeks on weekly applications. The dose of escitalopram used in the first period was $10 \mathrm{mg} / \mathrm{day}$, changing in the second step to $20 \mathrm{mg} /$ day. In a recent study (Moreno et al., 2020), the authors looked at the cognitive changes in the treatment groups, 
reporting a cognitive improvement in most tests for all experimental groups. The verbal fluency was significantly greater in the group receiving active TDCS, compared to placebo.

Furthermore, TDCS showed similar effects to $50 \mathrm{mg} /$ day sertraline in a recent clinical, randomized and controlled trial performed by Park and coworkers (2020) (Park et al., 2020). The study administered 12 home sessions of TDCS ( $2 \mathrm{~mA}$ current) with anode set at F3 and cathode set at F4 regions (bifrontal). In a recent systematic review (Fregni et al., 2020), the authors considered that the effectiveness of using anodic TDCS over DLPFC (F3) in improving MD is at A level of scientific evidence. The analyzed studies employed protocols with TDCS alone or combined with other therapeutic modalities, and concluded that associated techniques may improve the antidepressant TDCS-induced effects.

Therefore, considering the evidence that TDCS is effective for MD management, and the perspective of including this therapeutic modality among conventional treatments due to the few adverse effects and low cost (Berlim et al., 2009; Lee et al., 2017), our main objective was to evaluate the reported effectiveness of TDCS, combined or not with antidepressants, psychotherapy or cognitive therapies, on clinical and neuropsychological outcomes in MD patients, in order to answer the following question: "Is TDCS more effective alone or in combination with antidepressants, psychotherapy or cognitive therapies for the treatment of MD patients?"

\section{Methodology}

\subsection{Data sources and research}

We performed an integrative and retrospective review of clinical trials, guided by the PICO strategy (Figure 1), using the clinical and neuropsychological improvement as outcomes for comparison of individual or combined TDCS treatment.

Figure 1 - PICO strategy flowchart for the elaboration of the present research question.
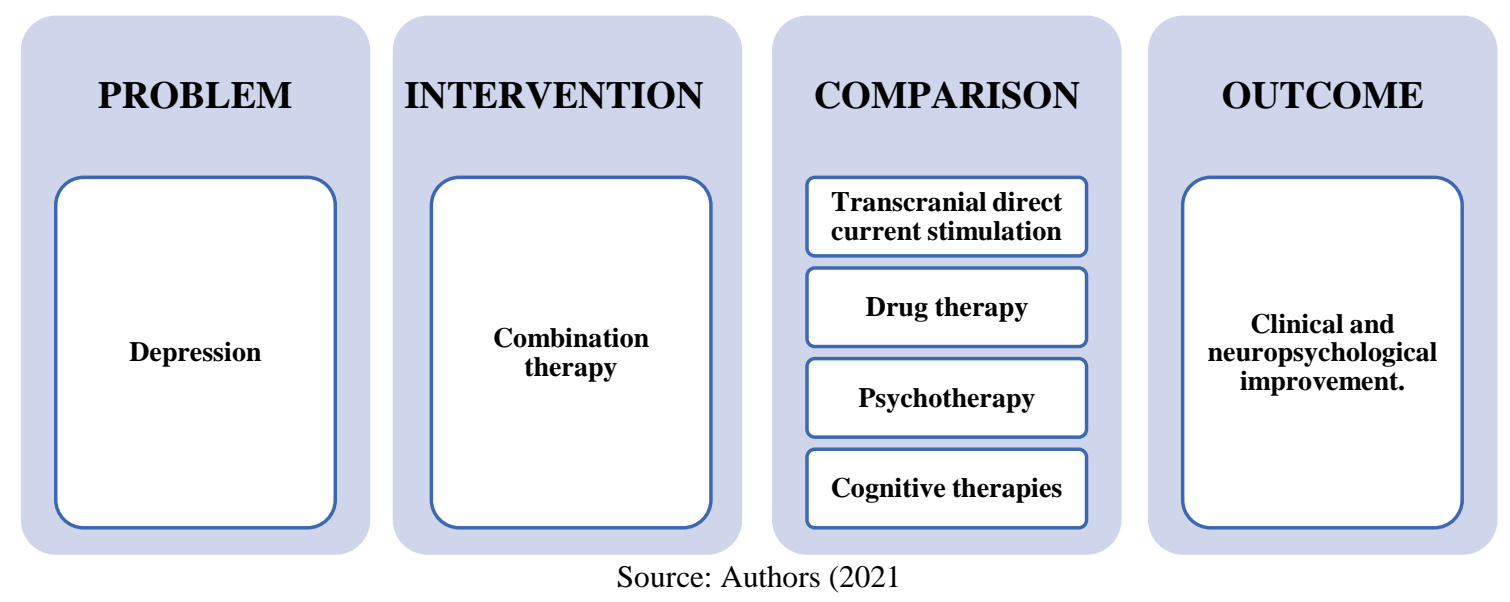

The present study employed the Ovid MEDLINE® and PubMed databases as search strategy. The full access of texts throughout the selection occurred through the PubMed platform. The complete search information can be found in SUPPLEMENTS.

\subsection{Study Selection}

Initially, duplicate articles were excluded. Afterwards, in a successive analysis oriented by titles, abstracts and full articles were selected according to the inclusion and exclusion criteria. 
As an inclusion criterion, only clinical studies were considered eligible for review. There was no limitation in relation to the year of publication.

As exclusion criteria, we considered studies which:

1) included in the sample individuals diagnosed with bipolar depression (bipolar affective disorder);

2) considered MD as a secondary disease;

3) included only partial results or contained only the abstract;

4) used other neurostimulation technique rather than TDCS;

5) investigated other therapies in addition to pharmacotherapy, psychotherapy and cognitive control therapy;

6) investigated the use of TDCS not combined and / or compared to the therapies listed in criterion 5;

7) analyzed the results after a single treatment session;

8) investigated non-clinical or neuropsychological outcomes.

The search and selection of studies took place during the month of July of 2021. Figure 2 shows the respective number of articles selected after each investigation step, culminating with the 12 reports on which the present review was based. 
Figure 2 - Steps for studies selection.

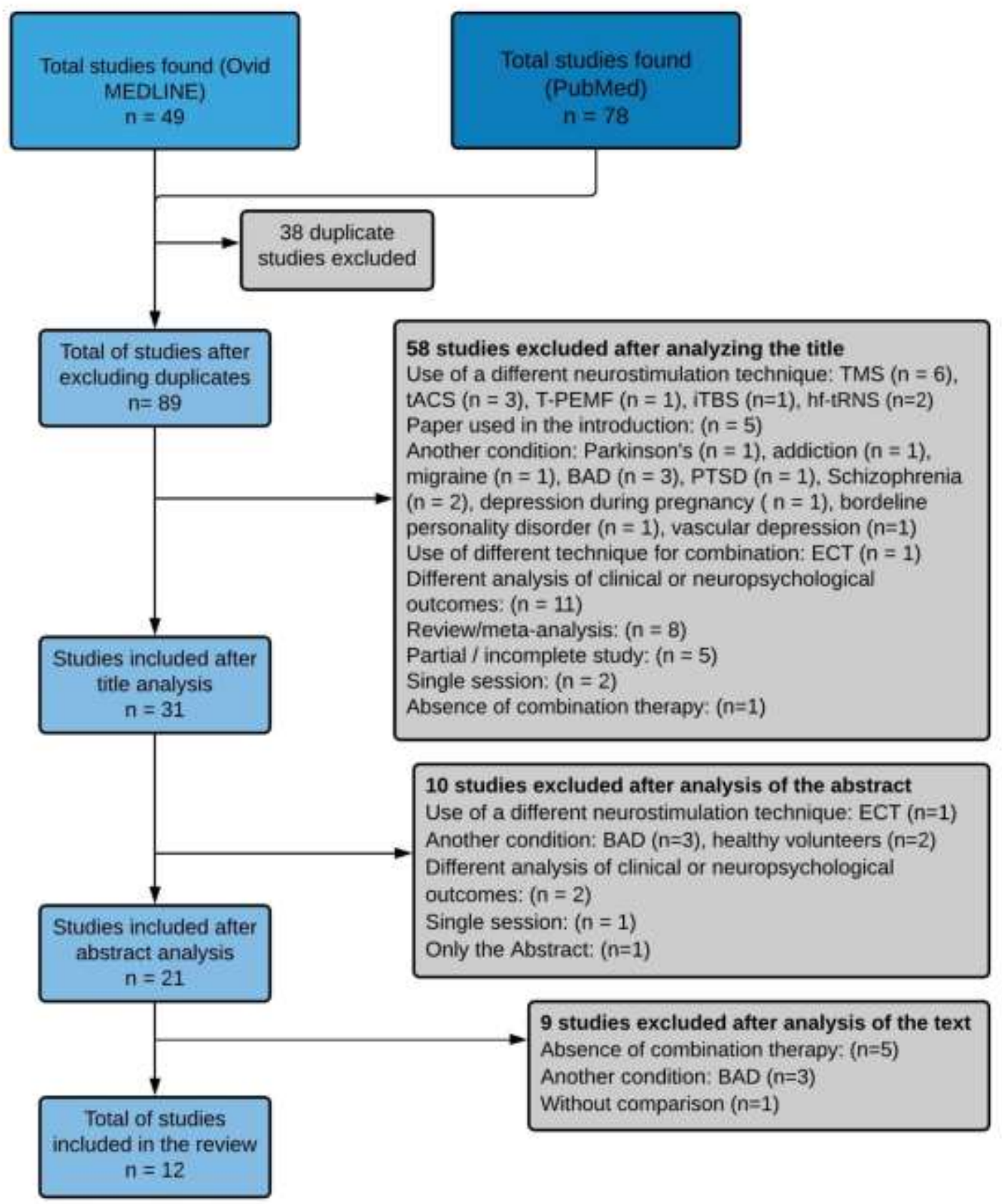

Note - TMS (Transcranial magnetic stimulation), tACS (Transcranial Alternating Current Stimulation), T-PEMF (transcranial pulsed eletromagnetic fields ), iTBS (Intermittent theta-burst stimulation ), hf-tRNS (high-frequency transcranial random noise stimulation ), BAD (bipolar affective disorder), PTSD (Post-traumatic stress disorder), ECT (Electroconvulsive therapy).

Source: Authors (2021).

\subsection{Analysis and Synthesis}

The articles included in the present review were categorized according to authors, year of publication, type of study, sample size, treatment groups, electrode arrangement, stimulation technique (current intensity, time and number of sessions) and main results.

In addition, we compared the studies to understand the implications and the relationship between their study methodologies, especially in relation to the techniques of neurostimulation and therapeutic association, and with their respective results, in an attempt to answer our research question and raise new scientific gaps to be considered for future studies. 


\section{Results and Discussion}

Tables 1, 2, 3 and 4 briefly present the selected studies and their specificities. In the following topics, we will try to address specific issues related to the efficacy of combined strategies for the management of MD, with emphasis on the use of TDCS and the comparison between methodological variables.

\subsection{Clinical efficacy of combined therapy:}

Of the seven studies in which comparison with the control group was possible, four demonstrated that the therapeutic combination was significantly more effective. Table 4 summarizes these studies, comparing the differences among them.

The two studies (Andre R Brunoni et al., 2013; Pavlova et al., 2018) which employed sertraline in association with TDCS showed positive results. Considering the two reports $(34,35)$ which analyzed the combined effects of TDCS with CCT, only Segrave and colleagues (Segrave et al., 2014) were able to demonstrate superior effectiveness of the combined treatment. However, these two reports showed relevant discrepancies in the methodological approach, particularly regarding electrode layout, time and number of sessions and protocol for CCT application.

The study that looked at the TDCS + CBT combination found no relevant difference in the analyzed outcomes when compared to CBT alone (Nord et al., 2019).

Finally, the analysis of TDCS effectiveness on patients undergoing refractory treatment with selective sertraline reuptake inhibitors (SSRIs) treatment was brought by two studies (Dell'Osso et al., 2014; Sharafi et al., 2019) with opposite results. Again, the final outcomes could have been impacted by methodological issues, such as different electrode arrangement, current intensity, as well as time and interval between sessions. 
Table 1 - Studies using association between transcranial direct current stimulation (TDCS) and cognitive-behavioral therapy (CBT) or TCDS and cognitive control training (CCT).

\begin{tabular}{|c|c|c|c|c|c|c|c|c|c|c|}
\hline Author & Year & Study & Sample & Groups & Disposition & $\begin{array}{l}\text { Current } \\
\text { Intensity }\end{array}$ & $\begin{array}{c}\text { Session } \\
\text { Time } \\
\end{array}$ & $\begin{array}{c}\text { Number of } \\
\text { sessions } \\
\end{array}$ & Association & Results \\
\hline Segrave et al. & 2014 & $\begin{array}{l}\text { Controlled } \\
\text { randomized } \\
\text { clinical trial } \\
\end{array}$ & 27 adults & $\begin{array}{l}\text { 1) } \mathrm{TDCS}+\mathrm{CCT} \\
\text { 2) sham + CCT } \\
\text { 3) } \mathrm{TDCS}+\text { sham }\end{array}$ & $\begin{array}{c}\text { Anode - F3 } \\
\text { Cathode - F8 }\end{array}$ & $2 \mathrm{~mA}$ & $24 \mathrm{~min}$ & $\begin{array}{c}5 \text { sessions } \\
1 / \text { day } \\
5 \text { days } \\
\end{array}$ & $\begin{array}{c}\text { Cognitive } \\
\text { controll training } \\
\text { simultaneous } \\
\end{array}$ & $\begin{array}{l}\text { Clinical and } \\
\text { cognitive } \\
\text { improvement }\end{array}$ \\
\hline $\begin{array}{c}\text { Brunoni et al. } \\
\text { \# }\end{array}$ & 2014 & $\begin{array}{l}\text { Controlled } \\
\text { randomized } \\
\text { clinical trial }\end{array}$ & 37 adults & $\begin{array}{l}\text { 1) sham + CCT } \\
\text { 2) } \mathrm{TDCS}+\mathrm{CCT}\end{array}$ & $\begin{array}{c}\text { Anode - F3 } \\
\text { Cathode - F4 }\end{array}$ & $2 \mathrm{~mA}$ & $30 \mathrm{~min}$ & $\begin{array}{c}10 \text { sessions } \\
5 / \text { week } \\
2 \text { weeks }\end{array}$ & $\begin{array}{c}\text { Cognitive } \\
\text { controll training } \\
\text { simultaneous }\end{array}$ & $\begin{array}{c}\text { No difference } \\
\text { between groups }\end{array}$ \\
\hline $\begin{array}{c}\text { Vanderhas selt et al. } \\
\text { \# }\end{array}$ & 2015 & & & & & & & & & $\begin{array}{c}\text { TDCS added } \\
\text { no addicional } \\
\text { effects } \\
\text { neuropsychological }\end{array}$ \\
\hline Khayyer et al. & 2018 & $\begin{array}{l}\text { Uncontrolled } \\
\text { randomized } \\
\text { clinical trial }\end{array}$ & 9 woman & $\begin{array}{l}\text { 1) TDCS } \\
\text { 2) } \mathrm{PP} \\
\text { 3) } \mathrm{TDCS}+\mathrm{PP}\end{array}$ & $\begin{array}{l}\text { Anode - F3 } \\
\text { or F4 } \\
\text { Cathode - Fz } \\
\text { or EC } \\
\end{array}$ & $1,5 \mathrm{~mA}$ & $15 \mathrm{~min}$ & $\begin{array}{c}12 \text { sessions } \\
\text { 3/week } \\
4 \text { weeks }\end{array}$ & $\begin{array}{c}\text { Positive } \\
\text { psychotherapy }\end{array}$ & $\begin{array}{c}\text { Clinical improvement } \\
\text { and hope and } \\
\text { otism } \\
\text { indicators } \\
\end{array}$ \\
\hline Welch et al. & 2019 & & 14 adults & $\begin{array}{l}\text { 1) } \mathrm{TDCS}+\mathrm{eCBT} \\
\text { 2) sham + eCBT }\end{array}$ & $\begin{array}{c}\text { Anode - F3 } \\
\text { Cathode - F4 }\end{array}$ & $2 \mathrm{~mA}$ & $30 \mathrm{~min}$ & $\begin{array}{c}12 \text { sessions } \\
\text { 3/week } \\
4 \text { weeks }\end{array}$ & $\begin{array}{c}\text { eCBT } \\
\text { simultaneous }\end{array}$ & $\begin{array}{l}\text { Symptom reduction } \\
\text { in both groups } \\
\text { comparison not } \\
\text { possible }\end{array}$ \\
\hline Nord et al. & 2019 & $\begin{array}{l}\text { Controlled } \\
\text { randomized } \\
\text { clinical trial }\end{array}$ & 33 adults & $\begin{array}{l}\text { 1) } \mathrm{TDCS}+\mathrm{CBT} \\
\text { 2) sham + CBT }\end{array}$ & $\begin{array}{c}\text { Anode - F3 } \\
\text { Cathode - ID }\end{array}$ & $1 \mathrm{~mA}$ & $20 \mathrm{~min}$ & $\begin{array}{l}8 \text { ses sões } \\
1 / \text { semana } \\
8 \text { semanas }\end{array}$ & $\begin{array}{c}\text { Cognitive } \\
\text { behavioral } \\
\text { therapy after }\end{array}$ & $\begin{array}{l}\text { No difference } \\
\text { between } \\
\text { groups }\end{array}$ \\
\hline
\end{tabular}

Note - TDCS (transcranial direct current stimulation), CCT (cognitive controll training), PP (positive psychotherapy), eCBT (eletronic cognitive-behavioral therapy), F3 (left front), F4 (right front), F8 (right previous temporal), Fz (midline frontal), EC (extra cephalic ), ID (ipslateral deltoid), (min (minute). \# They are complementary studies

Source: Authors (2021). 
Table 2 - Studies using association or comparison between transcranial direct current stimulation (TDCS) and pharmacotherapy.

\begin{tabular}{|c|c|c|c|c|c|c|c|c|c|c|}
\hline Author & Year & Study & Sample & Groups & Disposition & $\begin{array}{l}\text { Current } \\
\text { Intensity }\end{array}$ & $\begin{array}{c}\text { Session } \\
\text { Time } \\
\end{array}$ & $\begin{array}{c}\text { Number of } \\
\text { sessions }\end{array}$ & Association & Results \\
\hline $\begin{array}{c}\text { Brunoni et al. } \\
\text { \# }\end{array}$ & 2013 & $\begin{array}{l}\text { Controlled } \\
\text { randomized } \\
\text { clinical trial }\end{array}$ & 120 adults & $\begin{array}{l}\text { 1) Sham + placebo } \\
\text { 2) Sham + sertraline } \\
\text { 3) TDCS + placebo } \\
\text { 4) TDCS + sertraline }\end{array}$ & $\begin{array}{c}\text { Anode - F3 } \\
\text { Cathode - F4 }\end{array}$ & $2 \mathrm{~mA}$ & $30 \mathrm{~min}$ & $\begin{array}{c}12 \text { sessions } \\
5 / \text { week } \\
2 \text { weeks } \\
+ \\
1 / 15 \text { days } \\
2 \text { sessions }\end{array}$ & $\begin{array}{l}50 \mathrm{mg} \text { de } \\
\text { Sertraline }\end{array}$ & $\begin{array}{c}\text { TDCS better than placebo } \\
\text { it's equivalent to } \\
\text { sertraline } \\
\text { Superior combination therapy } \\
\text { and with answer } \\
\text { faster }\end{array}$ \\
\hline $\begin{array}{c}\text { Valiengo et al. } \\
\text { \# }\end{array}$ & 2013 & Clinical trial & 42 adults & TDCS & $\begin{array}{c}\text { Anode - F3 } \\
\text { Cathode - F4 }\end{array}$ & $2 \mathrm{~mA}$ & $30 \mathrm{~min}$ & $\begin{array}{c}1 / 15 \text { days } \\
\text { por } 3 \text { months } \\
1 / \text { month } \\
3 \text { months } \\
\end{array}$ & $\begin{array}{c}\text { Only } 8 \\
\text { maintained the } \\
\text { Sertraline }\end{array}$ & \\
\hline $\begin{array}{c}\text { Brunoni et al. } \\
\text { \# }\end{array}$ & 2016 & & & & & & & & & \\
\hline Pavlova et al. & 2018 & Clinical trial & 69 adults & $\begin{array}{l}\text { 1) TDCS + sertraline } \\
\text { 2) TDCS + sertraline } \\
\text { 3) Sham + sertraline }\end{array}$ & $\begin{array}{c}\text { Anode - F3 } \\
\text { Cathode - RSO }\end{array}$ & $0,5 \mathrm{~mA}$ & $\begin{array}{c}20 \text { or } \\
30 \mathrm{~min}\end{array}$ & $\begin{array}{c}10 \text { sessions } \\
5 / \text { week } \\
2 \text { weeks }\end{array}$ & $\begin{array}{l}50 \mathrm{mg} \text { de } \\
\text { Sertraline }\end{array}$ & $\begin{array}{l}\text { Clinical improvement } \\
\text { significantly higher } \\
\text { in the } 30 \text { and } 20 \text { min group. } \\
30 \text { min group with } \\
\text { improves significantly } \\
\text { greater than } 20 \text { min }\end{array}$ \\
\hline
\end{tabular}

Note - TDCS (transcranial direct current stimulation), F3 (left front), F4 (right front), RSO (right supra orbital), min (minute), mg (milligram). \# They are complementary studies.

Source: Authors (2021). 
Table 3 - Studies using association between transcranial direct current stimulation (TDCS) and refractory treatment with selective sertraline reuptake inhibitors (SSRIs).

\begin{tabular}{|c|c|c|c|c|c|c|c|c|c|c|}
\hline Author & Year & Study & Sample & Groups & Dis position & $\begin{array}{l}\text { Current } \\
\text { Intensity } \\
\end{array}$ & $\begin{array}{c}\text { Session } \\
\text { Time }\end{array}$ & $\begin{array}{c}\text { Number of } \\
\text { sessions } \\
\end{array}$ & Association & Results \\
\hline Bennabi et al & 2015 & $\begin{array}{l}\text { Controlled } \\
\text { randomized } \\
\text { clinical trial }\end{array}$ & 24 adults & $\begin{array}{l}\text { 1) TDCS } \\
\text { 2) sham }\end{array}$ & $\begin{array}{c}\text { Anode - F3 } \\
\text { Cathode - Fp2 }\end{array}$ & $2 \mathrm{~mA}$ & $30 \mathrm{~min}$ & $\begin{array}{c}10 \text { sessions } \\
2 / \text { day } \\
5 \text { days }\end{array}$ & $\begin{array}{c}\text { Es citalopram } \\
10 \text { a } 20 \mathrm{mg} \\
\text { fixed }\end{array}$ & $\begin{array}{c}\text { No signficant } \\
\text { difference } \\
\text { betwenn groups }\end{array}$ \\
\hline Sharafi et al. & 2019 & $\begin{array}{l}\text { Controlled } \\
\text { randomized } \\
\text { cinical trial }\end{array}$ & 30 adults & $\begin{array}{l}\text { 1) TDCS } \\
\text { 2) sham }\end{array}$ & $\begin{array}{c}\text { Anode - F3 } \\
\text { Cathode - F4 }\end{array}$ & $2 \mathrm{~mA}$ & $20 \mathrm{~min}$ & $\begin{array}{c}10 \text { sessions } \\
5 / \text { week } \\
2 \text { weeks }\end{array}$ & $\begin{array}{c}\text { Maintenance } \\
\text { of previus } \\
\text { SSRI }\end{array}$ & $\begin{array}{c}\text { TDCS more } \\
\text { significant than sham }\end{array}$ \\
\hline
\end{tabular}

Note - TDCS (transcranial direct current stimulation), F3 (left front), F4 (right front), Fp2 (right fronto-polar), min (minute), mg (milligram). Source: Authors (2021). 
Table 4 - Comparative analysis of protocol variables employed by the controlled clinical trials analyzed in the present study.

\begin{tabular}{|c|c|c|c|c|c|c|c|c|}
\hline & & & Intensity & Time & Between Sessions & sessions & & \\
\hline $\begin{array}{l}\text { TDCS } \\
+ \\
\text { CCT }\end{array}$ & $\begin{array}{l}\text { Segrave et al. (2014) } \\
\text { Brunoni et al. (2014) }\end{array}$ & $\mathrm{X}$ & & $\mathrm{X}$ & & $\mathrm{X}$ & $\mathrm{X}$ & Distint \\
\hline $\begin{array}{c}\text { TDCS } \\
+ \\
\text { Sertraline }\end{array}$ & $\begin{array}{l}\text { Brunoni et al. (2013) } \\
\text { Pavlova et al. (2018) }\end{array}$ & $\mathrm{X}$ & $\mathrm{X}$ & & & $\mathrm{X}$ & & Similar \\
\hline $\begin{array}{c}\text { Resistence } \\
\text { pharmachotherapy }\end{array}$ & $\begin{array}{l}\text { Bennabi et al. (2015) } \\
\text { Sharafi et al. (2019) }\end{array}$ & $\mathrm{X}$ & & $\mathrm{X}$ & $\mathrm{X}$ & & $\mathrm{X}$ & Distint \\
\hline TDCS & Segrave et al. (2014) & $\mathrm{F} 3 / \mathrm{F} 8$ & $2 \mathrm{~mA}$ & $24 \min$ & $1 /$ day & 5 & $\mathrm{CCT}(\mathrm{PSAT}+\mathrm{WAT})$ & + \\
\hline+ & & & & & & & & \\
\hline $\mathrm{CCT}$ & Brunoni et al. (2014) & $\mathrm{F} 3 / \mathrm{F} 4$ & $2 \mathrm{~mA}$ & $30 \mathrm{~min}$ & 1/day & 10 & CCT (PSAT) & - \\
\hline $\mathrm{TDCS}+\mathrm{CBT}$ & Nord et al. (2019) & F3/DI & $1 \mathrm{~mA}$ & $20 \mathrm{~min}$ & $1 /$ week & 8 & CBT & - \\
\hline TDCS & Brunoni et al. (2013) & $\mathrm{F} 3 / \mathrm{F} 4$ & $2 \mathrm{~mA}$ & $30 \mathrm{~min}$ & 1/day & 12 & $50 \mathrm{mg}$ s ertraline & + \\
\hline+ & & & & & & & & \\
\hline Sertraline & Pavlova et al. (2018) & F3/RSO & $0,5 \mathrm{~mA}$ & $30 \mathrm{~min}$ & 1/day & 10 & $50 \mathrm{mg}$ s ertraline & + \\
\hline Resistence & Bennabi et al. (2015) & $\mathrm{F} 3 / \mathrm{Fp} 2$ & $2 \mathrm{~mA}$ & $30 \mathrm{~min}$ & 2/day & 10 & $10-20 \mathrm{mg}$ escitalopram & - \\
\hline pharmachotherapy & Sharafi et al. (2019) & $\mathrm{F} 3 / \mathrm{F} 4$ & $2 \mathrm{~mA}$ & $20 \mathrm{~min}$ & $1 /$ day & 10 & SSRI & + \\
\hline
\end{tabular}

Note - F3 (left front), F4 (right front), F8 (right previous tempora ), Fp2 (right fronto-polar) ID (ipsilateral deltoid), RSO (right supra orbital ), min (minute), mg (miligram) CCT (cognitive controll training), CBT (cognitive-behavioral therapy), PSAT (Paced Serial Addition Task ), WAT (Wells Attentional Training ), SSRI (sertraline reuptake inhibitors)

The $\mathrm{X}$ indicates which variables were distinct between studies.

Source: Authors (2021). 


\subsection{Stimulation mode:}

We detected that all selected clinical trials involving MD patients used anodic stimulation positioned over the left prefrontal cortex, more specifically in F3 region, which corresponds to the DLPFC. Only Khayyer et al (2018) (Khayyer et al., 2018) also performed anodic stimulation in F4.

The involvement of ventromedial prefrontal cortex - vmPFC and DLPFC in MD pathogenesis is already known (Liu et al., 2017). The DLPFC, the targeted area of anodic stimulation in which the reports were concentrated, has a key function in cognitive functions such as working memory, attention, intention and action direction, whereas the vmPFC participates in emotional and affective functions (Koenigs \& Grafman, 2009; Liu et al., 2017).

MD is associated with DLPFC hypoactivity or injury, and vmPFC hyperactivity, so that the stimulation of DLPFC and a decreased vmPFC activity are related to a decrease in depressive mood (Koenigs \& Grafman, 2009). As mentioned above, MD patients also exhibit a prefrontal DLPFC asymmetry, with hypoactivity found on the left and hyperactivity on the right hemisphere (Grimm et al., 2008). This clinical finding is frequently restored after treatment (Grimm et al., 2008; Kocmur et al., 1998; Mottaghy et al., 2002), particularly due to a reduced activity on the left side (Bruder et al., 2017).

Studies performed on healthy individuals (Sanchez et al., 2016; Wolkenstein et al., 2014) corroborate the evidence of prefrontal asymmetry in depressive patients. Inhibition of the left DLPFC leads to altered processing of negative affect contents (Wolkenstein et al., 2014), whereas anodic stimulation of the right DLPFC in parallel with contralateral supraorbital cathode stimulation leads to an attention deficit (Sanchez et al., 2016). Thus, the application of anodic TDCS over left DLPFC (in association or not with the cathodic stimulation of the DLPFC) is supported by neuropathophysiological findings of the literature. However, it is important to mention that cathode positioning over brain regions other than the right DLPFC also produced responses.

The studies performed by Segrave et al. (2014) (Segrave et al., 2014), Brunoni et al. (2013) (Andre R Brunoni et al., 2013), Pavlova et al. (2018) (Pavlova et al., 2018) and Sharafi et al. (2019) (Sharafi et al., 2019) obtained significant therapeutic responses for combined therapy (TCDS plus pharmacotherapy or CCT), when compared to control groups. However, three reports used pharmacotherapy associated with anodic stimulation at F3 and cathodic stimulation at F4 (Andre R Brunoni et al., 2013; Sharafi et al., 2019) or right supra orbital region (SOR) (Pavlova et al., 2018), whereas Segrave and colleagues (2014) (Segrave et al., 2014) used the cathode positioned at F8 (right anterior temporal region).

All the studies in which the control groups were used, and which did not demonstrate positive results associated with the combined therapy, performed anodic stimulation at F3. Two studies (A R Brunoni et al., 2014; Vanderhasselt et al., 2015) that combined TDCS with CCT used the cathode positioned in F4; another report that combined TDCS with CBT, used an ipsilateral deltoid (ID) cathode; finally, Bennabi and coworkers (2015) employed TDCS in patients refractory to escitalopram treatment with the cathode arranged at Fp2 (right frontal region).

Therefore, we found a heterogeneity of cathode positioning, both for studies with significant results (F4, SOR, F8) and for those who found no significant outcomes (F4, ID, Fp2). This is a very important observation, particularly under the analysis of TDCS + CCT or TDCS + resistant pharmacotherapy associations, since the discrepant electrode disposition may certainly underlie the contrasting results.

Although it is difficult to draw a consistent conclusion about this variable, particularly due to the limited number of studies, we can establish some questions, which may help researchers to manage this issue in future studies: 
1) In the study developed by Segrave and colleagues (2014) (Segrave et al., 2014) (anode arrangement in F3 and cathode in F8), the effects of combined TDCS and CCT would remain the same if the cathodic stimulation had been performed at F4?

2) Using the same sample with anodic F3 stimulation, which arrangement would be the most effective (cathode at F4 or F8)?

3) Replicating the experimental design of Sharafi et al. (2019) (Sharafi et al., 2019), who employed TDCS in patients resistant to pharmacotherapy, would results be similar if electrodes were arranged at F3 (anode) and F4 (cathode)?

4) In MD patients resistant to pharmacotherapy and submitted to anodic TDCS at F3 region, is there a difference between the cathode arrangement in F4 and Fp2, in different groups of the same sample?

\subsection{Current intensity:}

Current intensity ranged from 0.5 to $2 \mathrm{~mA}$ in the analyzed studies. However, we found no correlation between the antidepressant response and this variable. Even in the study by Pavlova et al. (2018) (Pavlova et al., 2018), the stimulation of $0.5 \mathrm{~mA}$ combined with sertraline showed significant clinical improvement, being greater for the group with the longest stimulation time (30 minutes). Among the studies that used the highest current intensity ( $2 \mathrm{~mA}$ ), there were significant (Andre R Brunoni et al., 2013; Segrave et al., 2014; Sharafi et al., 2019) and non-significant results (Bennabi et al., 2015; A R Brunoni et al., 2014; Vanderhasselt et al., 2015). Nord et al. (2019) used an intermediate current intensity (1 mA) and also found no significant results when combining TDCS and CBT.

\subsection{Session time:}

The duration of each TDCS session varied from 15 to 30 minutes. Sessions lasting between 20 and 30 minutes were more effective in promoting significant results for combined therapy (Andre R Brunoni et al., 2013; Pavlova et al., 2018; Segrave et al., 2014; Sharafi et al., 2019). In this regard, we found an intriguing fact: among the studies in which TDCS was combined with CCT (A R Brunoni et al., 2014; Segrave et al., 2014) or with pharmacotherapy (Bennabi et al., 2015; Sharafi et al., 2019), two works showing positive results (Segrave et al., 2014; Sharafi et al., 2019) used shorter sessions (24 and 20 minutes, respectively) than those that did not report significant effect of the combined treatment.

In the study of Pavlova et al. (2018) (Pavlova et al., 2018), the session duration was positively correlated with clinical improvement. The group undergoing combined therapy was exposed to longer (30 minutes) TDCS stimulation, obtaining better results when compared to the group that underwent a 20-minute protocol and to the sham + sertraline group.

\subsection{Number of sessions and interval between sessions:}

The sessions varied widely in number, from 5 to 12. The distribution was bimodal, with three studies conducting 10 sessions, and four studies conducting 12 sessions. In fact, the study by Segrave et al. (2014) (Segrave et al., 2014) showed the effectiveness of the combined treatment (TDCS + CCT) in relation to the isolated treatments (TDCS + CCT sham the TDCS sham + CCT) after only 5 sessions (3 weeks of follow-up).

\subsection{Association of TDCS with CCT or CBT:}

In general, TDCS associated with CCT or CBT have shown contradictory results in the literature. The study by Segrave et al. (2014) (Segrave et al., 2014) was the only one that demonstrated potentiation of the antidepressant and cognitive in response 
to the combination of TDCS with cognitive techniques. The other studies, however, found no further improvement by TDCS. Although Khayyer and colleagues (2018) initially reported, for one of the groups, increased clinical improvement, as well as indicators of optimism and hope, in response to psychotherapy combined with TDCS, this study presents a critical selection bias, since its sample consisted only of women with a low number (3) of patients in each group, in addition to the lack of control group and the absence of statistical tests that could attest the significance of results.

The two studies that used presential (Nord et al., 2019) or virtual (Welch et al., 2019) CBT found no further improvement by TDCS. While Welch et al (2019)(Welch et al., 2019) performed 3 sessions / week and were unable to establish comparisons between groups due to the low number of volunteers, Nord et al (2019) (Nord et al., 2019) performed only 1 session / week / 8 weeks with CBT after TDCS (1 mA, 20 minutes).

Regarding the therapeutic modality most commonly found in the studies, CCT stands out in two studies: Segrave et al. (2014) (Segrave et al., 2014) and Brunoni et al. (2014) (A R Brunoni et al., 2014). Despite this, there was a difference in the experimental design between studies, with Segrave et al. (2014) (Segrave et al., 2014) being the only one to demonstrate the effectiveness of the therapeutic combination in a 3-week follow-up. These authors reported a response rate of $44 \%$ with only 5 sessions using a current intensity of $2 \mathrm{~mA}$ for 24 minutes, with anode disposed at F3 and cathode at F8. The other study used only one adapted Paced Serial Addition Task (PSAT), while Segrave et al. (2014) (Segrave et al., 2014) used two simultaneous cognitive tasks with TDCS, an adaptation of the PSAT and a modified Wells Attentional Training (WAT) paradigm.

Brunoni et al. (2014) (A R Brunoni et al., 2014), even without reporting a significant improvement, considered that the combined therapy tended to contribute to an overall increment in positive outcomes. However, when analyzing cognitive and clinical improvements comparatively among different ages, these authors found that in older patients (>50 years) TDCS increases the effects of CCT.

PSAT is based on arithmetic calculations in series of numbers presented by audio, in the face of a cognitively challenging situation. WAT, in turn, is designed to improve self-directed attention and control over automatic negative thoughts, being carried out by audio-guided tasks, in the face of constant distractions (Segrave et al., 2014). As already mentioned, DLPFC is critical for working memory and attention control, functions that are evaluated by the performance of both tasks.

It is already known that such cognitive tasks are related to functional recruitment of DLPFC and, therefore, circuits related to cognitive control, especially for negative information (Koster et al., 2017). Thus, TDCS concomitant with cognitive training activities may induce increased left DLPFC function, where the anode is positioned (F3 region).

The functional recruitment of DLPFC in these responses are also supported by in vitro studies performed by Rahman and group (2017) (Rahman et al., 2017), who reported an amplification of the electric field generated by neurostimulation in rodent brains, in a cooperative operation between the stimulated area and connected adjacent regions.

\subsection{Association with pharmacotherapy:}

In adult patients with mild to severe MD, studies have found more significant TDCS-mediated antidepressant effects with anodic position set at the DLPFC (F3) combined with $50 \mathrm{mg} /$ day of sertraline, when compared to control groups. These protocols used current intensities of 0.5 to $2 \mathrm{~mA}$ and 10 or 12 sessions of 30 minutes each. There were also significantly higher rates of response and remission when using combined therapy.

In a more recent study (Pavlova et al., 2018), which used the lowest electric current intensity (0,5 mA) applied to volunteers with mild and moderate MD, initially not treated, the combination of TDCS with sertraline was more effective when compared to the control group (sham $+50 \mathrm{mg}$ of sertraline) after 10 sessions. However, this effect varied in a time-dependent 
manner, so that the group with the longest session duration (30 minutes) obtained better results than the group with the shortest time exposure (20 minutes), which, in turn, obtained better results than the simulated stimulation. The response (89\%) and remission (70\%) also were only significant in the group with the longest protocol (30 min).

The study that showed the most significant results was the SELECT-TDCS survey (Andre R Brunoni et al., 2013), which involved 120 volunteers initially lacking antidepressant treatment, randomized into 4 groups: Group 1 - simulation of TDCS + oral placebo; Group 2 - simulation of TDCS + sertraline; Group 3 - active TDCS + oral placebo; Group 4 - Active TDCS + sertraline - with TDCS under bifrontal disposition (anode in F3 and cathode in F4), with 2 mA currents and 10 consecutive sessions of 30 minutes each, with 2 sessions of follow-up. A faster and more effective antidepressant effect was shown in the combination of therapies (50 mg of sertraline + active TDCS), as well as higher rates of response and remission, in addition to an equivalence of results between TDCS and sertraline and better results from the neurostimulation technique than placebo. The authors therefore considered that both treatments had additive effects.

In addition, Valiengo et al. (2013) (Valiengo et al., 2013) followed for 24 weeks (6 months) patients who presented antidepressant response in groups with active TDCS in phase I (Andre R Brunoni et al., 2013) and patients in phase II (nonresponders in groups with sham TDCS, who were subsequently submitted to 10 sessions of active TDCS with the same parameters as the SELECT-TDCS study). The sessions took place, in the first 3 months, every 2 weeks, and afterwards, once a month. The phase III participants presented the only statistically relevant difference, compared to the phase I, with a higher prevalence of melancholic depression and lower prevalence of atypical depression. Treatment-resistant depression was the only significant predictor of recurrence during follow-up. The use of sertraline (8 participants) had no significant association with recurrence and the mean duration of response was 11.7 weeks. However, the study had limitations due to the lack of a control group and a high dropout rate.

\subsection{Effect of TDCS on MD resistant to SSRI}

Two studies (Bennabi et al., 2015; Sharafi et al., 2019) investigated the effectiveness of TDCS for SSRI-resistant MD, and found divergent results.

Bennabi et al. (2015) (Bennabi et al., 2015), found no significant differences between the control (sham) and active groups, when evaluating the effectiveness of 10 sessions of TDCS, with administration of $2 \mathrm{~mA} / 30$ minutes, distributed over a week, with 2 sessions/day, with anode disposed at F3 and cathode at Fp2. The sample consisted of patients with resistance to escitalopram (dose between 10 and $20 \mathrm{mg} /$ day).

Unlike the previous findings, a recent study by Sharafi et al., 2019 (Sharafi et al., 2019) demonstrated that TDCS was significantly more effective than sham stimulation, maintaining this effect after 1 month. In this study, 10 TDCS sessions of 20 minutes each and 1 month of follow-up were applied to patients diagnosed with MD and resistant to SSRIs treatment. The authors employed $2 \mathrm{~mA}$ of current intensity and a bifrontal assembly (F3 and F4), with maintenance of the same SSRIs doses adopted prior to the study.

Although both studies used the same number of sessions (10), the most recent work (Sharafi et al., 2019), which showed more significant results of improvement in depressive symptoms in the group submitted to active TDCS, distributed the sessions throughout a longer period, in contrast to the previous study (Bennabi et al., 2015), in which the sessions were concentrated to occur in just 5 days. Together with different session durations (20 minutes for Sharafi et al., 2019 versus 30 minutes for Bennabi et al., 2015) and different medications used, the discrepant time-course protocol designs could explain the different outcomes. By the way, the drugs and their dosage were clearly defined only by the study by Bennabi et al. (2015) (Bennabi et al., 2015). 


\subsection{Results in neuropsychological indicators:}

Regarding neuropsychological results, the findings were contradictory and heterogeneous. Some studies showed that even with superior clinical improvement with combined treatment, there was no difference between groups when the improvement in neuropsychological tasks was analyzed (Andre Russowsky Brunoni et al., 2016). In contrast, others reported significant improvement for combined therapy in both clinical and neuropsychological parameters (Pavlova et al., 2018; Segrave et al., 2014). Finally, a third group of studies found significant increment in neurocognitive performance, but showed no significant clinical outcomes in response to combined therapy (A R Brunoni et al., 2014; Vanderhasselt et al., 2015).

In addition to the clinical improvement evidenced by Brunoni et al (2013), the cognitive effects of such interventions were also analyzed, and published in another study (Andre Russowsky Brunoni et al., 2016), with a general improvement of all treatment groups, regardless the therapeutic modality and clinical response, in most of the neuropsychological tasks applied at different time frames.

The study by Segrave et al. (2014) suggests that a combined therapy of TDCS + CCT may increase cognitive control over negative stimuli. In Pavlova's study, only one of the neuropsychological tests (Digit Span Backwards), which assesses working memory, showed a significant correlation, with both groups with active TDCS showing improvement in depressive mood. In the other tests, in all groups, the performance was better after treatment.

Brunoni et al. (2014) (A R Brunoni et al., 2014) found that there was a significant improvement in PSAT task performance in the combined group (TDCS + CCT), so even with an improvement in working memory performance, there was no additional antidepressant effect. Finally, in a complementary study (Vanderhasselt et al., 2015), the same group showed positive results in reducing ruminative thoughts of depressive content (as assed by PSAT), and the greater the improvement in this variable, the greater the improvement in working memory. There was no increase in effect by TDCS in the group with active stimulation.

In another study, also complementary to Brunoni et al. (A R Brunoni et al., 2014) and not included in the present review, the authors compared emotional reactivity among groups (Vanderhasselt et al., 2016) before and after interventions (CCT + sham TDCS or CCT + active TDCS) and its correlation with depressive symptoms. The study reported that a greater emotional reactivity for negative affect before the intervention (or less blunted reactivity) was associated with a greater reduction in depressive symptoms at the end of the study. However, the analysis referred only to the total sample of the original study, due to the depressive improvement in both groups.

In the work of Nord et al. (2019) (Nord et al., 2019), the active condition (TDCS + CBT) was associated with a significant increase in activity of the right posterior parietal cortex during working memory task, and with increased activity of left DLPFC during emotional processing. In addition, pretreatment activation of left DLPFC was significantly associated with symptomatic improvement in the active condition, with volunteers with high activation of left DLPFC being more likely to respond to TDCS. Initial working memory, however, was not a good predictive for clinical improvement.

\subsection{Summary:}

The studies that show significant effects for depressive symptoms improvement with combined therapy were carried out with anodic stimulations at DLPFC (F3), with the cathode arrangement that varied between 3 different assemblies (F4, F8 and SOR) according to the associated therapeutic modality. Intensity current ranged from $0.5 \mathrm{~mA}$ to $2 \mathrm{~mA}$ (most often), with 5 to 12 sessions (mean of 10 sessions) lasting for 20 to 30 minutes, employing more commonly as therapeutic associations with TDCS 
the CCT (with 2 tasks - PSAT and WAT) and SSRIs treatment (with emphasis on sertraline). Tables 5 and 6 summarize significant and non-significant results.

Table 5 - Clinical studies showing greater effectiveness of combined therapy.

\begin{tabular}{ccccccc}
\hline Author & Disposition & $\begin{array}{c}\text { Current } \\
\text { Intensity }\end{array}$ & $\begin{array}{c}\text { Session } \\
\text { Time }\end{array}$ & $\begin{array}{c}\text { Interval } \\
\text { Between Sessions }\end{array}$ & $\begin{array}{c}\text { Number of } \\
\text { sessions }\end{array}$ & Association \\
\hline Segrave et al. (2014) & F3/F8 & $2 \mathrm{~mA}$ & $24 \mathrm{~min}$ & $1 /$ day & 5 & CCT (PSAT + WAT) \\
\hline Brunoni et al. (2013) & F3/F4 & $2 \mathrm{~mA}$ & $30 \mathrm{~min}$ & $1 /$ day +2 additional & 12 & $50 \mathrm{mg}$ of Sertraline \\
\hline Pavlova et al. (2018) & F3/RSO & $0,5 \mathrm{~mA}$ & $30 \mathrm{~min}$ & $1 /$ day & 10 & $50 \mathrm{mg}$ of Sertraline \\
\hline Sharafi et al. (2019) & F3/F4 & $2 \mathrm{~mA}$ & $20 \mathrm{~min}$ & $1 /$ day & 10 & SSRI \\
\hline
\end{tabular}

Note - F3 (left front), F4 (right front), F8 (right previous tempora), RSO (right supra orbital), min (minute), mg (miligram), CCT (cognitive controll training), PSAT (Paced Serial Addition Task), WAT (Wells Attentional Training), SSRI (sertraline reuptake inhibitors)

Source: Authors (2021).

Table 6 - Clinical studies that have not found statistical relevant results of combined therapy.

\begin{tabular}{ccccccrc}
\hline Author & Disposition & $\begin{array}{c}\text { Current } \\
\text { Intensity }\end{array}$ & $\begin{array}{c}\text { Session } \\
\text { Time }\end{array}$ & $\begin{array}{c}\text { Interval } \\
\text { Between Sessions }\end{array}$ & $\begin{array}{c}\text { Number of } \\
\text { sessions }\end{array}$ & Association \\
\hline Brunoni et al. (2014) & F3/F4 & $2 \mathrm{~mA}$ & $30 \mathrm{~min}$ & $1 /$ day & 10 & CCT (PSAT) \\
\hline Nord et al. (2019) & F3/DI & $1 \mathrm{~mA}$ & $20 \mathrm{~min}$ & $1 /$ week & 8 & CBT \\
\hline Bennabi et al. (2015) & F3/Fp2 & $2 \mathrm{~mA}$ & $30 \mathrm{~min}$ & $2 /$ day & 10 & $10-20 \mathrm{mg}$ Es citalopram \\
\hline
\end{tabular}

Note - F3 (left front), F4 (right front ), Fp2 (right fronto-polar ), ID (ipsilateral deltoid ), min (minute), mg (miligram), CCT (cognitive controll training ), PSAT (Paced Serial Addition Task), CBT (cognitive-behavioral therapy )

Source: Authors (2021).

\subsection{Future perspectives:}

Although the study by Brunoni et al. (2013) (Andre R Brunoni et al., 2013) demonstrated superior effectiveness of the combined treatment of TDCS with sertraline in relation to the isolated therapy (sertraline or TDCS), more studies comparing and combining other antidepressant drugs with TDCS would provide further evidence on the reliability of this therapeutic modality.

In addition, the divergent results between the studies that combined CCT with TDCS can be better clarified with additional studies, using larger samples. Furthermore, the study design employed by Segrave and colleagues (2014) (Segrave et al., 2014), in which two tools (PSAT and WAT) were used for CCT, would better help future studies to answer the key question: Is the combination of CCT and TDCS more effective that therapy alone?

Considering that some studies have not established comparisons with the control group - because they do not had it or because of the small sample, and due to the lack of uniformity between the methods, some unanswered issues still remain:

1) Is CBT associated with TDCS more effective than alone?

2) Is positive psychotherapy combined with TDCS more effective than alone?

Moreover, regarding the use of TDCS in patients resistant to pharmacological antidepressant therapy with SSRIs, further studies with a larger number of volunteers should be carried out to confirm the most recent results by Sharafi et al. (2019) (Sharafi 
et al., 2019). Lastly, if possible and feasible, studies with a longer follow-up time would better verify the long-term effects of the combined treatment when compared to isolated therapies.

\section{Conclusion}

Based on the set of studies evaluated and evidence already present from other studies, anodic TDCS applied to the DLPFC has significant antidepressant effects for the management of MD. Such effects may be similar or discreetly inferior than those obtained in response to antidepressant drugs commonly used in clinical practice, but superior to placebo or simulated stimulation. Furthermore, the studies analyzed indicate that the antidepressant therapeutic effects can be enhanced by the combination of TDCS mainly with sertraline, even at low doses (50 mg/day). Cognitive function may be also enhanced by TDCS, although more studies, with a larger number of volunteers, are still needed in order to better support this hypothesis. Further studies may also better support the correlation between neurocognitive and clinical improvement with this type of combined therapy.

Thus, unclear questions regarding the use of TDCS in association with other therapies still remain, particularly due to the few existing studies, distinct research methods, low number of volunteers and short follow-up time. New studies should better explore the synergism between CBT and ETCC, also expanding comparisons with other antidepressants and other psychotherapeutic modalities.

\section{References}

Arul-Anandam, A. P., \& Loo, C. (2009). Transcranial direct current stimulation: a new tool for the treatment of depression? Journal of Affective Disorders, 117(3), 137-145. https://doi.org/10.1016/j.jad.2009.01.016

Association, A. P. (2014). Manual Diagnóstico e Estatístico de Transtornos Mentais (5 ed.). ARTMED.

Bennabi, D., Nicolier, M., Monnin, J., Tio, G., Pazart, L., Vandel, P., \& Haffen, E. (2015). Pilot study of feasibility of the effect of treatment with tDCS in patients suffering from treatment-resistant depression treated with escitalopram. Clinical Neurophysiology: Official Journal of the International Federation of Clinical Neurophysiology, 126(6), 1185-1189. https://doi.org/10.1016/j.clinph.2014.09.026

Berlim, M. T., Dias Neto, V., \& Turecki, G. (2009). [Transcranial direct current stimulation: a promising alternative for the treatment of major depression?]. Revista Brasileira de Psiquiatria (Sao Paulo, Brazil : 1999), 31 Suppl 1(Suppl I), S34-8. https://doi.org/10.1590/S1516-44462009000500006

Berti de Azevedo Barros, M. I., Guimarães Lima, M. I., Cruz Soares de Azevedo, R. I., Barbosa de Paula Medina III, L., de Souza Lopes, C. I., Rossi Menezes, P. V, Carvalho Malta, D. V., \& A Barros Rua Tessália Vieira de Camargo, M. B. (2016). DESCRIPTORS: Depressive Disorder, epidemiology. Health Behavior. Health Knowledge, Attitudes, Practice. Health Surveys. 1-9. http://www.rsp.fsp.usp.br/2s

Bruder, G. E., Stewart, J. W., \& McGrath, P. J. (2017). Right brain, left brain in depressive disorders: Clinical and theoretical implications of behavioral, electrophysiological and neuroimaging findings. Neuroscience and Biobehavioral Reviews, 78, 178-191. https://doi.org/10.1016/j.neubiorev.2017.04.021

Brunoni, A R, Boggio, P. S., De Raedt, R., Bensenor, I. M., Lotufo, P. A., Namur, V., Valiengo, L. C. L., \& Vanderhasselt, M. A. (2014). Cognitive control therapy and transcranial direct current stimulation for depression: a randomized, double-blinded, controlled trial. Journal of Affective Disorders, 162, 43-49. https://doi.org/https://dx.doi.org/10.1016/j.jad.2014.03.026

Brunoni, Andre R, Moffa, A. H., Sampaio-Junior, B., Borrione, L., Moreno, M. L., Fernandes, R. A., Veronezi, B. P., Nogueira, B. S., Aparicio, L. V. M., Razza, L. B., Chamorro, R., Tort, L. C., Fraguas, R., Lotufo, P. A., Gattaz, W. F., Fregni, F., Bensenor, I. M., \& Investigators, E.-T. (2017). Trial of Electrical DirectCurrent Therapy versus Escitalopram for Depression. The New England Journal of Medicine, 376(26), 2523-2533. https://doi.org/https://dx.doi.org/10.1056/NEJMoa1612999

Brunoni, Andre R, Valiengo, L., Baccaro, A., Zanao, T. A., de Oliveira, J. F., Goulart, A., Boggio, P. S., Lotufo, P. A., Bensenor, I. M., \& Fregni, F. (2013). The sertraline vs. electrical current therapy for treating depression clinical study: results from a factorial, randomized, controlled trial. JAMA Psychiatry, 70(4), 383-391. https://doi.org/https://dx.doi.org/10.1001/2013.jamapsychiatry.32

Brunoni, Andre Russowsky, Tortella, G., Bensenor, I. M., Lotufo, P. A., Carvalho, A. F., \& Fregni, F. (2016). Cognitive effects of transcranial direct current stimulation in depression: Results from the SELECT-TDCS trial and insights for further clinical trials. Journal of Affective Disorders, 202 , 46-52. https://doi.org/https://dx.doi.org/10.1016/j.jad.2016.03.066

Çakir, S., \& Çağlar, N. (2017). Electroconvulsive Therapy in the Treatment of Mood Disorders: One-Year Follow-up. Noro Psikiyatri Arsivi, 54(3), 196-201. https://doi.org/10.5152/npa.2016.14845 
Csifcsák, G., Boayue, N. M., Puonti, O., Thielscher, A., \& Mittner, M. (2018). Effects of transcranial direct current stimulation for treating depression: A modeling study. Journal of Affective Disorders, 234, 164-173. https://doi.org/10.1016/j.jad.2018.02.077

Cuijpers, P., Berking, M., Andersson, G., Quigley, L., Kleiboer, A., \& Dobson, K. S. (2013). A meta-analysis of cognitive-behavioural therapy for adult depression, alone and in comparison with other treatments. Canadian Journal of Psychiatry. Revue Canadienne de Psychiatrie, 58(7), 376-385. https://doi.org/10.1177/070674371305800702

Dean, J., \& Keshavan, M. (2017). The neurobiology of depression: An integrated view. Asian Journal of Psychiatry, 27, 101-111. https://doi.org/10.1016/j.ajp.2017.01.025

Dell'Osso, B., Dobrea, C., Arici, C., Benatti, B., Ferrucci, R., Vergari, M., Priori, A., \& Altamura, A. C. (2014). Augmentative transcranial direct current stimulation (tDCS) in poor responder depressed patients: a follow-up study. CNS Spectrums, 19(4), 347-354. https://doi.org/https://dx.doi.org/10.1017/S1092852913000497

Depression-Global-Health-Estimates @Www.Who.Int. (n.d.). https://www.who.int/publications/i/item/depression-global-health-estimates

Edwards, C. A., Kouzani, A., Lee, K. H., \& Ross, E. K. (2017). Neurostimulation Devices for the Treatment of Neurologic Disorders. Mayo Clinic Proceedings, 92(9), 1427-1444. https://doi.org/10.1016/j.mayocp.2017.05.005

Fregni, F., El-Hagrassy, M. M., Pacheco-Barrios, K., Carvalho, S., Leite, J., Simis, M., Brunelin, J., Nakamura-Palacios, E. M., Marangolo, P., Venkatasubramanian, G., San-Juan, D., Caumo, W., Bikson, M., \& Brunoni, A. R. (2020). Evidence-based guidelines and secondary meta-analysis for the use of transcranial direct current stimulation (tDCS) in neurological and psychiatric disorders. The International Journal of Neuropsychopharmacology. https://doi.org/10.1093/ijnp/pyaa051

Grimm, S., Beck, J., Schuepbach, D., Hell, D., Boesiger, P., Bermpohl, F., Niehaus, L., Boeker, H., \& Northoff, G. (2008). Imbalance between left and right dorsolateral prefrontal cortex in major depression is linked to negative emotional judgment: an fMRI study in severe major depressive disorder. Biological Psychiatry, 63(4), 369-376. https://doi.org/10.1016/j.biopsych.2007.05.033

Hermida, A. P., Glass, O. M., Shafi, H., \& McDonald, W. M. (2018). Electroconvulsive Therapy in Depression: Current Practice and Future Direction. The Psychiatric Clinics of North America, 41(3), 341-353. https://doi.org/10.1016/j.psc.2018.04.001

Hillhouse, T. M., \& Porter, J. H. (2015). A brief history of the development of antidepressant drugs: from monoamines to glutamate. Experimental and Clinical Psychopharmacology, 23(1), 1-21. https://doi.org/10.1037/a0038550

Khayyer, Z., Ngaosuvan, L., Sikstrom, S., \& Ghaderi, A. H. (2018). Transcranial direct current stimulation based on quantitative electroencephalogram combining positive psychotherapy for major depression. Journal of Integrative Neuroscience. https://doi.org/https://dx.doi.org/10.31083/JIN-170045

Kim, Y.-K., \& Won, E. (2017). The influence of stress on neuroinflammation and alterations in brain structure and function in major depressive disorder. Behavioural Brain Research, 329, 6-11. https://doi.org/10.1016/j.bbr.2017.04.020

Kocmur, M., Milcinski, M., \& Budihna, N. V. (1998). Evaluation of brain perfusion with technetium-99m bicisate single-photon emission tomography in patients with depressive disorder before and after drug treatment. European Journal of Nuclear Medicine, 25(10), 1412-1414. https://doi.org/10.1007/s002590050316

Koenigs, M., \& Grafman, J. (2009). The functional neuroanatomy of depression: distinct roles for ventromedial and dorsolateral prefrontal cortex. Behavioural Brain Research, 201(2), 239-243. https://doi.org/10.1016/j.bbr.2009.03.004

Koster, E. H. W., Hoorelbeke, K., Onraedt, T., Owens, M., \& Derakshan, N. (2017). Cognitive control interventions for depression: A systematic review of findings from training studies. Clinical Psychology Review, 53, 79-92. https://doi.org/10.1016/j.cpr.2017.02.002

Lee, J. C., Lewis, C. P., Daskalakis, Z. J., \& Croarkin, P. E. (2017). Transcranial Direct Current Stimulation: Considerations for Research in Adolescent Depression. Frontiers in Psychiatry, 8, 91. https://doi.org/10.3389/fpsyt.2017.00091

Lefaucheur, J.-P., Antal, A., Ayache, S. S., Benninger, D. H., Brunelin, J., Cogiamanian, F., Cotelli, M., De Ridder, D., Ferrucci, R., Langguth, B., Marangolo, P., Mylius, V., Nitsche, M. A., Padberg, F., Palm, U., Poulet, E., Priori, A., Rossi, S., Schecklmann, M., ... Paulus, W. (2017). Evidence-based guidelines on the therapeutic use of transcranial direct current stimulation (tDCS). Clinical Neurophysiology: Official Journal of the International Federation of Clinical Neurophysiology, 128(1), 56-92. https://doi.org/10.1016/j.clinph.2016.10.087

Liu, W., Ge, T., Leng, Y., Pan, Z., Fan, J., Yang, W., \& Cui, R. (2017). The Role of Neural Plasticity in Depression: From Hippocampus to Prefrontal Cortex. Neural Plasticity, 2017, 6871089. https://doi.org/10.1155/2017/6871089

López-López, J. A., Davies, S. R., Caldwell, D. M., Churchill, R., Peters, T. J., Tallon, D., Dawson, S., Wu, Q., Li, J., Taylor, A., Lewis, G., Kessler, D. S., Wiles, N., \& Welton, N. J. (2019). The process and delivery of CBT for depression in adults: a systematic review and network meta-analysis. Psychological Medicine, 49(12), 1937-1947. https://doi.org/10.1017/S003329171900120X

Mennen, A. C., Norman, K. A., \& Turk-Browne, N. B. (2019). Attentional bias in depression: understanding mechanisms to improve training and treatment. Current Opinion in Psychology, 29, 266-273. https://doi.org/10.1016/j.copsyc.2019.07.036

Moreno, M. L., Goerigk, S. A., Bertola, L., Suemoto, C. K., Razza, L. B., Moffa, A. H., Veronezi, B. P., Tort, L., Nogueira, B. S., Gattaz, W. F., Fraguas, R., Padberg, F., Lotufo, P. A., Benseñor, I. M., \& Brunoni, A. R. (2020). Cognitive changes after tDCS and escitalopram treatment in major depressive disorder: Results from the placebo-controlled ELECT-TDCS trial. Journal of Affective Disorders, 263, 344-352. https://doi.org/10.1016/j.jad.2019.12.009 
Mottaghy, F. M., Keller, C. E., Gangitano, M., Ly, J., Thall, M., Parker, J. A., \& Pascual-Leone, A. (2002). Correlation of cerebral blood flow and treatment effects of repetitive transcranial magnetic stimulation in depressed patients. Psychiatry Research, 115(1-2), 1-14. https://doi.org/10.1016/s09254927(02)00032-x

Nord, C. L., Halahakoon, D. C., Limbachya, T., Charpentier, C., Lally, N., Walsh, V., Leibowitz, J., Pilling, S., \& Roiser, J. P. (2019). Neural predictors of treatment response to brain stimulation and psychological therapy in depression: a double-blind randomized controlled trial. Neuropsychopharmacology : Official Publication of the American College of Neuropsychopharmacology, 44(9), 1613-1622. https://doi.org/https://dx.doi.org/10.1038/s41386-019-0401-0

Park, S., Choi, W.-J., Kim, S., Kim, B., Son, S. J., Roh, D., Kim, W. J., \& Park, J. Y. (2020). Effects of transcranial direct current stimulation using miniaturized devices vs sertraline for depression in Korea: A 6 week, multicenter, randomized, double blind, active-controlled study. Journal of Psychiatric Research, 127, 42-47. https://doi.org/10.1016/j.jpsychires.2020.04.012

Pavlova, E. L., Menshikova, A. A., Semenov, R. V, Bocharnikova, E. N., Gotovtseva, G. N., Druzhkova, T. A., Gersamia, A. G., Gudkova, A. A., \& Guekht, A. B. (2018). Transcranial direct current stimulation of 20- and 30-minutes combined with sertraline for the treatment of depression. Progress in NeuroPsychopharmacology \& Biological Psychiatry, 82, 31-38. https://doi.org/https://dx.doi.org/10.1016/j.pnpbp.2017.12.004

Rahman, A., Lafon, B., Parra, L. C., \& Bikson, M. (2017). Direct current stimulation boosts synaptic gain and cooperativity in vitro. The Journal of Physiology, 595(11), 3535-3547. https://doi.org/10.1113/JP273005

Sanchez, A., Vanderhasselt, M.-A., Baeken, C., \& De Raedt, R. (2016). Effects of tDCS over the right DLPFC on attentional disengagement from positive and negative faces: An eye-tracking study. Cognitive, Affective \& Behavioral Neuroscience, 16(6), 1027-1038. https://doi.org/10.3758/s13415-016-0450-3

Santoft, F., Axelsson, E., Öst, L.-G., Hedman-Lagerlöf, M., Fust, J., \& Hedman-Lagerlöf, E. (2019). Cognitive behaviour therapy for depression in primary care: systematic review and meta-analysis. Psychological Medicine, 49(8), 1266-1274. https://doi.org/10.1017/S0033291718004208

Segrave, R. A., Arnold, S., Hoy, K., \& Fitzgerald, P. B. (2014). Concurrent cognitive control training augments the antidepressant efficacy of tDCS: a pilot study. Brain Stimulation, 7(2), 325-331. https://doi.org/https://dx.doi.org/10.1016/j.brs.2013.12.008

Sharafi, E., Taghva, A., Arbabi, M., Dadarkhah, A., \& Ghaderi, J. (2019). Transcranial Direct Current Stimulation for Treatment-Resistant Major Depression: A Double-Blind Randomized Sham-Controlled Trial. Clinical EEG and Neuroscience, 50(6), 375-382. https://doi.org/https://dx.doi.org/10.1177/1550059419863209

Swedish Council on Health Technology Assessment. (2004). Treatment of Depression: A Systematic Review.

Valiengo, L., Bensenor, I., Goulart, A., de Oliveira, J., Zanao, T., Boggio, P., Lotufo, P., Fregni, F., \& Brunoni, A. (2013). THE SERTRALINE VERSUS ELECTRICAL CURRENT THERAPY FOR TREATING DEPRESSION CLINICAL STUDY (SELECT-TDCS): RESULTS OF THE CROSSOVER AND FOLLOW-UP PHASES. Depress. Anxiety, 30(7), 646-653. https://doi.org/10.1002/da.22079

Vanderhasselt, M.-A., De Raedt, R., Namur, V., Lotufo, P., Bensenor, I., Boggio, P., \& Brunoni, A. (2015). Transcranial electric stimulation and neurocognitive training in clinically depressed patients: A pilot study of the effects on rumination. Prog. Neuro-Psychopharmacol. Biol. Psychiatry, 57, 93-99. https://doi.org/10.1016/j.pnpbp.2014.09.015

Vanderhasselt, M.-A., De Raedt, R., Namur, V., Valiengo, L. C. L., Lotufo, P. A., Bensenor, I. M., Baeken, C., Boggio, P. S., \& Brunoni, A. R. (2016). Emotional reactivity to valence-loaded stimuli are related to treatment response of neurocognitive therapy. Journal of Affective Disorders, 190, 443-449. https://doi.org/https://dx.doi.org/10.1016/j.jad.2015.10.022

Welch, E., Weigand, A., Hooker, J., Philip, N., Tyrka, A., MD, P., Press, D., \& Carpenter, L. (2019). Feasibility of Computerized Cognitive-Behavioral Therapy Combined With Bifrontal Transcranial Direct Current Stimulation for Treatment of Major Depression. Neuromodulation, 22(8), 898-903. https://doi.org/10.1111/ner.12807

Willner, P., Scheel-Krüger, J., \& Belzung, C. (2013). The neurobiology of depression and antidepressant action. Neuroscience and Biobehavioral Reviews, 37(10 Pt 1), 2331-2371. https://doi.org/10.1016/j.neubiorev.2012.12.007

Wolkenstein, L., Zeiller, M., Kanske, P., \& Plewnia, C. (2014). Induction of a depression-like negativity bias by cathodal transcranial direct current stimulation. Cortex; a Journal Devoted to the Study of the Nervous System and Behavior, 59, 103-112. https://doi.org/10.1016/j.cortex.2014.07.011

Zakhour, S., Nardi, A. E., Levitan, M., \& Appolinario, J. C. (2020). Cognitive-behavioral therapy for treatment-resistant depression in adults and adolescents: a systematic review. Trends in Psychiatry and Psychotherapy, 42(1), 92-101. https://doi.org/10.1590/2237-6089-2019-0033 


\section{SUPPLEMENTS:}

\section{PubMed (04/07/2021):}

15. (\#8 OR \#9 OR \#10 OR \#11) Filters: Clinical Trial, Randomized Controlled Trial, Humans

14. (\#8 OR \#9 OR \#10 OR \#11) Filters: Clinical Trial, Humans

13. (\#8 OR \#9 OR \#10 OR \#11) Filters: Humans

12. (\#8 OR \#9 OR \#10 OR \#11)

11. (\#1 AND \#2 AND \#6 AND \#7)

10. (\#1 AND \#2 AND \#5 AND \#7)

9. (\#1 AND \#2 AND \#4 AND \#7)

8. (\#1 AND \#2 AND \#3 AND \#7)

7. (RANDOMIZED CONTROLLED TRIALS AS TOPIC) OR (CLINICAL TRIALS, RANDOMIZED) OR (TRIALS, RANDOMIZED CLINICAL) OR (CONTROLLED CLINICAL TRIALS, RANDOMIZED) OR (((CLINICAL[TITLE/ABSTRACT] AND TRIAL[TITLE/ABSTRACT]) OR CLINICAL TRIALS AS TOPIC[MESH TERMS] OR CLINICAL TRIAL[PUBLICATION TYPE] OR RANDOM*[TITLE/ABSTRACT] OR RANDOM ALLOCATION[MESH TERMS] OR THERAPEUTIC USE[MESH SUBHEADING])) OR ((RANDOMIZED CONTROLLED TRIAL[PUBLICATION TYPE] OR (RANDOMIZED[TITLE/ABSTRACT] AND CONTROLLED[TITLE/ABSTRACT] AND TRIAL[TITLE/ABSTRACT])))

6. (COMBINED MODALITY THERAPY) OR (MULTIMODAL TREATMENT) OR (MULTIMODAL TREATMENTS) OR (TREATMENT, MULTIMODAL) OR (TREATMENTS, MULTIMODAL) OR (THERAPY, COMBINED MODALITY) OR (COMBINED MODALITY THERAPIES) OR (MODALITY THERAPIES, COMBINED) OR (MODALITY THERAPY, COMBINED) OR (THERAPIES, COMBINED MODALITY)

5. (Psychotherapy) OR (Psychotherapies)

4.(COGNITIVE BEHAVIORAL THERAPY) OR (BEHAVIORAL THERAPIES, COGNITIVE) OR (BEHAVIORAL THERAPY, COGNITIVE) OR (COGNITIVE BEHAVIORAL THERAPIES) OR (THERAPIES, COGNITIVE BEHAVIORAL) OR (THERAPY, COGNITIVE BEHAVIORAL) OR (THERAPY, COGNITIVE BEHAVIOR) OR (COGNITIVE BEHAVIOR THERAPY) OR (COGNITIVE THERAPY) OR (BEHAVIOR THERAPY, COGNITIVE) OR (BEHAVIOR THERAPIES, COGNITIVE) OR (COGNITIVE BEHAVIOR THERAPIES) OR (THERAPIES, COGNITIVE BEHAVIOR) OR (COGNITIVE PSYCHOTHERAPY) OR (COGNITIVE PSYCHOTHERAPIES) OR (PSYCHOTHERAPIES, COGNITIVE) OR (PSYCHOTHERAPY, COGNITIVE) OR (THERAPY, COGNITIVE) OR (COGNITIVE THERAPIES) OR (THERAPIES, COGNITIVE) OR (COGNITION THERAPY) OR (THERAPY, COGNITION) OR (COGNITION THERAPIES) OR (THERAPIES, COGNITION)

3. (DRUG THERAPY) OR (THERAPY, DRUG) OR (DRUG THERAPIES) OR (THERAPIES, DRUG) OR (PHARMACOTHERAPY) OR (PHARMACOTHERAPIES)

2. (TRANSCRANIAL DIRECT CURRENT STIMULATION) OR (TDCS) OR (CATHODAL STIMULATION TRANSCRANIAL DIRECT CURRENT STIMULATION) OR (CATHODAL STIMULATION TDCS) OR (CATHODAL STIMULATION TDCSS) OR (STIMULATION TDCS, CATHODAL) OR (STIMULATION TDCSS, CATHODAL) OR (TDCS, CATHODAL STIMULATION) OR (TDCSS, CATHODAL STIMULATION) OR (TRANSCRANIAL ELECTRICAL STIMULATION) OR (ELECTRICAL STIMULATION, TRANSCRANIAL) OR (ELECTRICAL STIMULATIONS, TRANSCRANIAL) OR (STIMULATION, TRANSCRANIAL ELECTRICAL) OR (STIMULATIONS, TRANSCRANIAL ELECTRICAL) OR (TRANSCRANIAL ELECTRICAL STIMULATIONS) OR (ANODAL STIMULATION TRANSCRANIAL DIRECT CURRENT STIMULATION) OR (ANODAL STIMULATION TDCS) OR (ANODAL STIMULATION TDCSS) OR (STIMULATION TDCS, ANODAL) OR (STIMULATION TDCSS, ANODAL) OR (TDCS, ANODAL STIMULATION) OR (TDCSS, ANODAL STIMULATION) OR (REPETITIVE TRANSCRANIAL ELECTRICAL STIMULATION)

1. (Depressive Disorder) OR (Depressive Disorders) OR (Disorder, Depressive) OR (Disorders, Depressive) OR (Depressive Syndrome) OR (Depressive Syndromes) OR (Syndrome, Depressive) OR (Syndromes, Depressive) OR (Unipolar Depression) OR (Depression, Unipolar) OR (Depressions, Unipolar) OR (Unipolar Depressions)

\section{Ovid (MEDLINE) (04/07/2021):}

1. (depressive disorder and transcranial direct current stimulation and drug therapy).af.

2. (depressive disorder and transcranial direct current stimulation and cognitive therapy).af.

3. (depressive disorder and transcranial direct current stimulation and combined modality therapy).af.

4. (depressive disorder and transcranial direct current stimulation and psychotherapy).af.

5. 1 or 2 or 3 or 4

6. limit 5 to humans

7. limit 6 to "therapy (best balance of sensitivity and specificity) 